\title{
UMIDADE DE EQUILÍBRIO DE PAINÉIS OSB PRODUZIDOS COM INCLUSÃO LAMINAR E COM DIFERENTES TIPOS DE ADESIVOS
}

\author{
Lourival Marin Mendes ${ }^{1}$, Rafael Farinassi Mendes ${ }^{1 *}$, Thiago de Paula Protásioํㅡ, \\ Stefânia Lima Oliveira' ${ }^{1}$, Ricardo Gabriel de Almeida Mesquita ${ }^{1}$
}

*Autor para correspondência: rafaelfarinassi@gmail.com

RESUMO: Neste trabalho, objetivou-se avaliar diferentes modelos estatísticos para a estimativa da umidade de equilíbrio de painéis OSB expostos a diferentes condições de temperatura e umidade relativa do ar, bem como avaliar a influência do adesivo e do reforço laminar na umidade de equilíbrio. Os painéis foram produzidos com três diferentes tipos de adesivos (fenol-formaldeído - FF, melamina-uréia-formaldeído - MUF, e fenol-melamina-uréia-formaldeído - PMUF) e, ainda, com e sem a inclusão laminar. A avaliação da umidade de equilíbrio dos painéis foi efetuada nas temperaturas de 30,40 e $50^{\circ} \mathrm{C}$ e nas umidades relativas de 40 , $50,60,70,80$ e $90 \%$. A modelagem da umidade de equilíbrio foi realizada por meio do ajuste de modelos estatísticos não lineares e polinomiais. De forma geral, os modelos polinomiais são os mais indicados para a estimativa da umidade de equilíbrio dos painéis OSB. Os modelos ajustados apenas com a umidade relativa do ar foram os que obtiveram as melhores medidas de precisão. O tipo de adesivo afetou a umidade de equilíbrio dos painéis, sendo observada para os adesivos PMUF e FF a mesma tendência de variação, e os maiores valores médios obtidos para os painéis produzidos com adesivo MUF. A inclusão laminar promoveu a diminuição da umidade de equilíbrio apenas para os painéis produzidos com o adesivo MUF.

Palavras-chave: Umidade de equilíbrio, modelagem, temperatura, umidade relativa do ar.

\section{EQUILIBRIUM MOISTURE CONTENT OF OSB PANELS PRODUCED WITH VENEER INCLUSION AND DIFFERENT TYPES OF ADHESIVE}

\begin{abstract}
The aim of this study was to evaluate different statistical models to estimate the equilibrium moisture content of OSB panels exposed to different conditions of air temperature and relative humidity, And also to evaluate the influence of the adhesive and veneer inclusion in the equilibrium moisture content. The panels were produced with three different adhesive types (phenolformaldehyde-FF, melamine-urea-formaldehyde-MUF, and phenol-melamine-urea-formaldehyde - PMUF) and with and without veneer inclusion. The evaluation of the equilibrium moisture content of the panels was carried out at temperatures of 30 , 40 and $50^{\circ} \mathrm{C}$ and relative humidity of 40,50,60,70,80 and 90\%. The modeling of equilibrium moisture content was performed using the statistical non-linear and polynomial models. In general, the polynomial models are most indicated for determining the equilibrium moisture content of OSB. The models adjusted only with air relative humidity presented the best precision measurements. The type of adhesive affected the equilibrium moisture content of the panels, being observed for adhesives PMUF and FF the same trend of variation, and the highest values obtained for the panels produced with adhesive MUF. The veneer inclusion decreased the equilibrium moisture content only in the panels with MUF adhesive.
\end{abstract}

Key words: Equilibrium moisture content, modeling, temperature, relative humidity of air.

\section{INTRODUÇÃO}

Os painéis OSB, por serem constituídos de madeira, um material higroscópico, quando em contato com o ar, absorvem ou perdem água no estado líquido ou de vapor até atingir um equilíbrio, onde essas trocas se equivalem e a umidade da madeira tende a se estabilizar (MARTINS et al., 2003).

As variações referentes a essa perda ou ganho de umidade, tem grande importância para o uso adequado de cada painel, já que deformações referentes a essa reação ao contato com a umidade são indesejáveis e apresentam relação direta com as demais propriedades dos painéis,

\footnotetext{
${ }^{1}$ Universidade Federal de Lavras - Lavras, Minas Gerais, Brasil
}

podendo vir a afetar, de forma significativa, a resistência mecânica e a estabilidade dimensional dos mesmos (SILVA et al., 2005).

As equações utilizadas para a predição da umidade de equilíbrio da madeira foram baseadas nas teorias de sorção de Hailwood e Harrobin, Bradley e BET descritas por Skaar (1972), sendo que Simpson (1971, 1973) e Simpson e Rosen (1981) determinaram os coeficientes originais das equações baseadas nessas teorias.

A equação mais utilizada para a predição da umidade de equilíbrio é aquela baseada na teoria de Hailwood e Harrobin e, alguns autores, como Mendes e Arce (2003), vêm empregando-a para estimar a umidade

Cerne, Lavras, v. 20, n. 1, p. 123-138, jan./mar. 2014 
de equilíbrio da madeira em várias localidades no Brasil.

A equação de Nelson (1983) ajustada por Wu (1999), baseada na energia livre de Gibbs, também é utilizada para a estimativa da umidade de equilíbrio de produtos à base de madeira (MENDES et al., 2004; SILVA et al., 2005, 2006b). No entanto, Silva et al. (2005) observaram tendência de subestimativa dos valores de umidade de equilíbrio de painéis de madeira obtidos por meio dessa equação.

Essas equações, apesar de serem utilizadas para a estimativa da umidade de equilíbrio, além de mais complexas, não foram desenvolvidas para painéis de madeira reconstituída que apresentam, geralmente, menor umidade de equilíbrio em relação à madeira maciça, em razão da maior densificação (SILVA et al., 2006b), emprego de temperatura durante a produção, aplicação de repelente de água e adesivos e pré-secagem da madeira.

Para uma maior precisão dessa estimativa devese proceder a um estudo de determinação prática das umidades de equilíbrio reais da madeira, bem como dos produtos de madeira, no seu local de uso por meio da utilização de uma equação que se aproxime da realidade (MENDES et al., 2004).

Nesse contexto, deve-se priorizar o uso de modelos estatísticos ajustados para cada tipo de painel, levando-se em consideração as condições específicas de sua produção. Os modelos de regressão permitem a estimativa indireta da umidade de equilíbrio, facilitando, assim, a compreensão e estudo dessa importante variável mediante as variações climáticas locais, principalmente a umidade relativa do ar e a temperatura.

Logo, a determinação da umidade de equilíbrio dos diferentes produtos de madeira, dentre os quais os painéis
OSB, em diferentes temperaturas e umidades relativas do ar, é de grande utilidade na busca do entendimento do comportamento dos painéis em um local específico.

Sendo assim, objetivou-se, neste trabalho, avaliar a influência do tipo de adesivo e do reforço laminar sobre a umidade de equilíbrio de painéis OSB, expostos a diferentes condições de temperatura e umidade relativa do ar, bem como ajustar modelos de regressão para a estimativa indireta da umidade de equilíbrio.

\section{MATERIAL E MÉTODOS}

\subsection{Matéria-prima}

Os painéis OSB foram confeccionados com a madeira de Pinus taeda L., com idade de 30 anos e procedentes de um plantio homogêneo localizado na Estação Experimental do Cangüiri da Universidade Federal do Paraná (UFPR), município de Pinhais-PR.

\subsection{Delineamento experimental}

O delineamento experimental se constituiu de seis tratamentos, conforme descrito na Tabela 1. Sendo avaliado o efeito de três diferentes tipos de adesivos (FF, PMUF e MUF) e também do reforço laminar dos painéis. Para cada tratamento, foram produzidos quatro painéis com dimensões de 48 x 48 x 1,5 cm (comprimento x largura $\mathrm{x}$ espessura), densidade nominal de $0,65 \mathrm{~g} / \mathrm{cm}^{3}$ e dimensões das partículas "strands" de 85 × 25 x 0,7 mm (comprimento, largura e espessura respectivamente).

Tabela 1 - Delineamento experimental para a produção dos painéis OSB.

Table 1 - Experimental design for the production of OSB.

\begin{tabular}{ccc}
\hline Tratamento & Adesivo & Reforço laminar \\
\hline 1 & Fenol-formaldeído (FF) & - \\
2 & Fenol-melamina-uréia-formaldeído (PMUF) & - \\
3 & Melamina-uréia-formaldeído (MUF) & L-P-L \\
4 & Fenol-formaldeído (FF) & L-P-L \\
6 & Fenol-melamina-uréia-formaldeído (PMUF) & L-P-L \\
\hline
\end{tabular}

L-P-L: Lâmina-Painel-Lâmina

Cerne, Lavras, v. 20, n. 1, p. 123-138, jan./mar. 2014 


\subsection{Manufatura dos painéis}

Para a produção dos painéis OSB, foram utilizados 6\% adesivo (Com base no teor de sólidos), conforme tratamentos pré-estabelecidos, sendo a aplicação realizada por meio de aspersão em uma encoladeira do tipo tambor giratório. As características dos três tipos de adesivos utilizados estão apresentadas na Tabela 2. A avaliação dos valores de $\mathrm{pH}$, teor de sólidos e viscosidade seguiu o procedimento da norma ASTM D1582 (AMERICAN SOCIETY FOR TESTING AND MATERIALS - ASTM, 1994).

Tabela 2 - Características dos adesivos utilizados na produção dos painéis OSB.

Table 2 - Characteristics of the adhesives used in the production of $O S B$.

\begin{tabular}{cccc}
\hline Adesivo & $\mathrm{pH}$ & Teor de sólidos (\%) & Viscosidade (cP) \\
\hline FF & 12,0 & 45,0 & 220 \\
PMUF & 9,5 & 60,7 & 200 \\
MUF & 7,5 & 66,2 & 210 \\
\hline
\end{tabular}

Após o encolamento das partículas "strand", estas foram distribuídas sobre uma caixa orientadora, conforme proporções em massa, estabelecidas para cada camada, as quais foram de $25 \%, 50 \%$ e $25 \%$ (face $/$ miolo/ face, respectivamente), sendo o miolo disposto de forma perpendicular as faces.

Na produção dos tratamentos com inclusão laminar, foram utilizadas lâminas de $2 \mathrm{~mm}$ de espessura também da espécie Pinus taeda. Para cada lâmina foram aplicados 160 $\mathrm{g} / \mathrm{cm}^{2}$ de linha de cola, sendo o adesivo fenol-formaldeído utilizado na colagem das lâminas de todos os tratamentos. Cabe ressaltar, que as lâminas com adesivo foram incorporadas no sentido perpendicular às partículas da face do painel, durante o processo de formação do colchão e prensadas simultaneamente (Figura 1).

Foi realizada uma pré-prensagem, com o intuito de proporcionar uma melhor conformação do painel e evitar a perda de partículas. Posteriormente, realizou-se prensagem, com ciclo de 8 minutos, pressão de $4 \mathrm{MPa}$ e temperaturas de $160^{\circ} \mathrm{C}$ para os painéis produzidos com o adesivo MUF e de $180^{\circ} \mathrm{C}$ para os produzidos com os adesivos FF e PMUF.

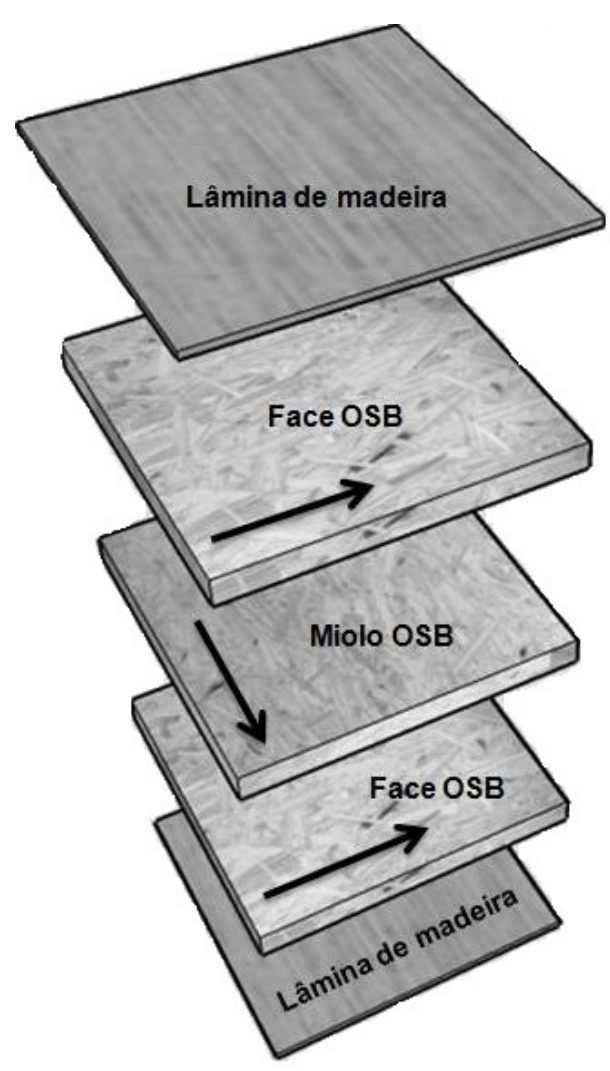

Figura 1 - Esquema de inclusão laminar.

Figure 1-Schematic of venner inclusion.

\subsection{Determinação da umidade de equilíbrio}

Para cada tratamento foram retirados 24 corpos de prova, sendo seis para cada painel. As dimensões dos corpos de prova foram de $25 \times 25 \mathrm{~mm}$, conforme metodologia desenvolvida por $\mathrm{Wu}$ (1999).

Os corpos de prova foram divididos em três grupos de oito amostras, sendo cada grupo levado para uma câmara de climatização regulada com temperatura de 30,40 e $50^{\circ} \mathrm{C}$, onde se efetuou a variação da umidade relativa (40, $50,60,70,80$ e 90\%). Após a estabilização dos corpos de prova, em cada uma das condições de temperatura e umidade relativa do ar, foi efetuada a determinação da massa dos mesmos. Ao final, todas as amostras foram levadas para uma estufa com temperatura de $103 \pm 5^{\circ} \mathrm{C}$, o que permitiu o registro da massa seca de cada corpo de

Cerne, Lavras, v. 20, n. 1, p. 123-138, jan./mar. 2014 
prova e, por consequência, proporcionou a determinação da umidade de equilíbrio das amostras em cada condição de temperatura e umidade.

\subsection{Análises estatísticas}

A modelagem da umidade de equilíbrio foi realizada por meio do ajuste de um modelo estatístico não linear (Eq.1) e um polinomial de terceira ordem (Eq. 2), considerando-se as temperaturas de 30 e $40^{\circ} \mathrm{C}$ para cada tratamento e como variável independente a umidade relativa do ar.

$$
\begin{aligned}
& U E Q=\beta_{0}+\beta_{1} e^{\beta 2^{*} U R}+\varepsilon \\
& U E Q=\beta_{0}+\beta_{1} U R+\beta_{2} U R^{2}+\beta_{3} U R^{3}+\varepsilon
\end{aligned}
$$

Em que: $U E Q$ é a umidade de equilíbrio dos painéis (\%); $\beta_{0,} \beta_{1}$ e $\beta_{2}$ são os parâmetros do modelo; $e$ é a base do logaritmo neperiano; UR é a umidade relativa do ar (\%) e $\varepsilon$ é o erro do modelo.

Já, para a temperatura de $50^{\circ} \mathrm{C}$ não foi possível obter o ajuste dos modelos descritos nas Eq. 1 e 2, sendo necessário considerar o modelo não linear e o polinômio de segunda ordem, representados nas Eq. $3 \mathrm{e}$ 4 , respectivamente.

$$
\begin{aligned}
& U E Q=\beta_{1} e^{\beta 2^{*} U R}+\varepsilon \\
& U E Q=\beta_{0}+\beta_{1} U R+\beta_{2} U R^{2}+\varepsilon
\end{aligned}
$$

Os melhores modelos foram avaliados com base na significância de todos os seus coeficientes (teste t a $5 \%$ de significância), no erro padrão da estimativa, no coeficiente de determinação ajustado, na presença de normalidade residual (teste Shapiro-Wilk) e com base no critério de informação de Akaike (AIC) e no critério de informação Bayesiano (BIC). Tanto o AIC quanto o BIC aumentam conforme SQE aumenta. Além disso, ambos os critérios penalizam modelos com muitas variáveis, sendo que valores menores de AIC e BIC são preferíveis. Além disso, para os modelos polinomiais foram realizadas análises de variância a 5\% de significância pelo Teste $\mathrm{F}$.

Cerne, Lavras, v. 20, n. 1, p. 123-138, jan./mar. 2014
Também foram ajustados modelos polinomiais múltiplos (superfícies de resposta), considerando como variáveis independentes a umidade relativa e a temperatura média do ar, conforme a Eq. 5.

$\mathrm{UEQ}=\beta_{0}+\beta_{1} \mathrm{UR}+\beta_{2} \mathrm{TEMP}+\beta_{3} \mathrm{UR}^{*} \mathrm{TEMP}+\beta_{4} \mathrm{UR}^{2}+$ $+\beta_{5}$ TEMP $^{2}+\varepsilon$

Onde: $U E Q$ é a umidade de equilíbrio (\%); UR é a umidade relativa do ar (\%); TEMP é a temperatura média do $\operatorname{ar}\left({ }^{\circ} \mathrm{C}\right) ; \beta_{0}, \beta_{1}, \beta_{2}, \beta_{3}, \beta_{4}$ e $\beta_{5}$ são os coeficientes do modelo e $\varepsilon$ é o erro do modelo.

Para as superfícies de resposta, foram realizadas análises de variância. Além disso, avaliou-se a significância dos coeficientes, o erro padrão da estimativa (Sxy), a normalidade dos resíduos (teste Shapiro-Wilk) e os critérios de informação de Akaike e o Bayesiano. Com as equações de superfície ajustadas para cada tratamento, foram determinados os pontos críticos (pontos estacionários) e sua classificação, ou seja, se o ponto é de máximo, mínimo ou sela, segundo as diretrizes de Myers (1971).

\section{RESULTADOS E DISCUSSÃO}

Na Tabela 3, são apresentados os parâmetros dos modelos não lineares ajustados com as diferentes umidades relativas, considerando a temperatura média de $30^{\circ} \mathrm{C}$, bem como algumas estatísticas utilizadas na avaliação da qualidade do ajuste.

Observa-se que a variabilidade referente à umidade de equilíbrio dos painéis OSB pode ser explicada pela umidade relativa do ar. Com exceção do modelo ajustado para o tratamento 3 , os demais modelos não lineares apresentaram, de maneira geral, todos os coeficientes significativos e baixo erro padrão da estimativa. Dessa forma, se pode inferir que os dados observados estão próximos da curva média ajustada.

Nota-se ainda que os modelos ajustados para os tratamentos 3 e 4 não apresentaram normalidade residual. Para construir testes de hipóteses ou intervalos de confiança, é preciso que o modelo apresente normalidade residual. Além disso, desvios em relação à normalidade podem influenciar a estatística comprometendo as inferências 
relacionadas ao modelo (WERKEMA; AGUIAR, 2006).

$\mathrm{Na}$ Tabela 4, encontram-se os parâmetros dos modelos polinomiais de terceira ordem ajustados considerando-se como variável independente a umidade relativa, considerando-se a temperatura média de $30^{\circ} \mathrm{C}$, bem como algumas estatísticas utilizadas na avaliação da qualidade do ajuste. Observou-se que todos os modelos polinomiais foram significativos pelo Teste $F(p<0,01)$.

Tabela 3 - Parâmetros dos modelos não lineares ajustados considerando a temperatura média de $30^{\circ} \mathrm{C}$.

Table 3 - Parameters of the nonlinear models adjusted considering the average temperature of $30^{\circ} \mathrm{C}$.

\begin{tabular}{|c|c|c|c|c|c|c|c|c|}
\hline \multirow[t]{2}{*}{ Tratamentos } & \multicolumn{2}{|c|}{ Coeficientes } & \multirow{2}{*}{$\frac{\text { tc }}{23,50^{* *}}$} & \multirow[t]{2}{*}{$\mathrm{R}_{\text {ajust }}^{2}$} & \multirow[t]{2}{*}{$S_{x y}$} & \multirow[t]{2}{*}{$\mathrm{AIC}$} & \multirow[t]{2}{*}{$\mathrm{BIC}$} & \multirow[t]{2}{*}{$\mathrm{W}$} \\
\hline & $\beta_{0}$ & 6,164 & & & & & & \\
\hline \multirow[t]{3}{*}{1} & $\beta_{1}$ & 0,023 & $2,52^{*}$ & 0,980 & 0,73 & 111,47 & 118,96 & $0,9715^{\mathrm{ns}}$ \\
\hline & $\beta_{2}$ & 0,072 & $16,67^{* *}$ & & & & & \\
\hline & $\beta_{0}$ & 5,014 & $11,57^{* *}$ & & & & & \\
\hline \multirow[t]{3}{*}{2} & $\beta_{1}$ & 0,079 & $1,83^{\mathrm{ns}}$ & 0,998 & 0,75 & 84,36 & 90,58 & $0,9752^{\mathrm{ns}}$ \\
\hline & $\beta_{2}$ & 0,056 & $9,58^{* *}$ & & & & & \\
\hline & $\beta_{0}$ & 12,134 & $2,72^{*}$ & & & & & \\
\hline \multirow[t]{3}{*}{3} & $\beta_{1}$ & 0,002 & $0,02^{\mathrm{ns}}$ & 0,787 & 8,01 & 249,85 & 256,07 & $0,9225^{*}$ \\
\hline & $\beta_{2}$ & 0,094 & $0,13^{\mathrm{ns}}$ & & & & & \\
\hline & $\beta_{0}$ & 6,319 & $23,87^{*}$ & & & & & \\
\hline \multirow[t]{3}{*}{4} & $\beta_{1}$ & 0,011 & $2,24^{\mathrm{ns}}$ & 0,998 & 0,84 & 124,81 & 132,30 & $0,9509^{*}$ \\
\hline & $\beta_{2}$ & 0,081 & $16,59^{*}$ & & & & & \\
\hline & $\beta_{0}$ & 5,467 & $18,05^{* *}$ & & & & & \\
\hline \multirow[t]{3}{*}{5} & $\beta_{1}$ & 0,021 & $1,95^{\mathrm{ns}}$ & 0,998 & 0,82 & 112,50 & 119,64 & $0,9537^{\mathrm{ns}}$ \\
\hline & $\beta_{2}$ & 0,072 & $12,83^{* *}$ & & & & & \\
\hline & $\beta_{0}$ & 4,856 & $15,82^{* *}$ & & & & & \\
\hline \multirow[t]{2}{*}{6} & $\beta_{1}$ & 0,024 & $2,07^{*}$ & 0,998 & 0,82 & 119,30 & 126,70 & $0,9638^{\mathrm{ns}}$ \\
\hline & $\beta_{2}$ & 0,071 & $13,49^{* *}$ & & & & & \\
\hline
\end{tabular}

tc : t calculado; $\mathrm{R}^{2}$ ajust: coeficiente de determinação ajustado; AIC: critério de informação de Akaike; BIC: critério de informação Bayesiano; $\mathrm{S}_{\mathrm{xy}}$ : erro padrão da estimativa (\%); W: estimativa do parâmetro do teste de normalidade Shapiro-Wilk; **: significativo a $1 \%$; *: significativo a $5 \%$; ns: não significativo a $5 \%$.

Observa-se que, assim como observado para o modelo não linear (Tabela 3), não foi possível obter um bom ajuste do polinômio de terceira ordem para o tratamento 3 , resultando, assim, em um elevado erro padrão da estimativa e ausência de normalidade residual. Os demais modelos ajustados apresentaram todos os coeficientes altamente significativos, alto coeficiente de determinação ajustado e normalidade residual.

Analisando os critérios de informação de Akaike
(AIC) e o critério de informação Bayesiano (BIC) pode-se observar que os modelos polinomiais de terceira ordem são os mais recomendados para a estimativa indireta da umidade de equilíbrio de painéis OSB considerando-se os tratamentos avaliados na temperatura média do ar de $30^{\circ} \mathrm{C}$. A representação gráfica da umidade de equilíbrio dos painéis estimada pelos modelos ajustados com as diferentes umidades relativas, considerando a temperatura de $30^{\circ} \mathrm{C}$, encontra-se na Figura 2.

Cerne, Lavras, v. 20, n. 1, p. 123-138, jan./mar. 2014 
Tabela 4 - Parâmetros dos modelos polinomiais de terceira ordem ajustados considerando a temperatura média de $30^{\circ} \mathrm{C}$.

Table 4 - Parameters of the third-order polynomial models adjusted, considering the average temperature of $30^{\circ} \mathrm{C}$.

\begin{tabular}{|c|c|c|c|c|c|c|c|c|}
\hline Tratamentos & & entes & tc & $\mathrm{R}_{\text {ajust }}^{2}$ & $S_{x y}$ & AIC & $\mathrm{BIC}$ & $\mathrm{W}$ \\
\hline \multirow{4}{*}{1} & $\beta_{0}$ & $-54,50$ & $-10,38^{* *}$ & \multirow{4}{*}{0,991} & \multirow{4}{*}{0,48} & \multirow{4}{*}{72,16} & \multirow{4}{*}{81,52} & \multirow{4}{*}{$0,9802^{\text {ns }}$} \\
\hline & $\beta_{1}$ & 3,19 & $12,26^{* *}$ & & & & & \\
\hline & $\beta_{2}$ & $-5,51.10^{-2}$ & $-13,22^{* *}$ & & & & & \\
\hline & $\beta_{3}$ & $3,21.10^{-4}$ & $15,15^{* *}$ & & & & & \\
\hline \multirow{4}{*}{2} & $\beta_{0}$ & $-32,38$ & $-3,96^{* *}$ & \multirow{4}{*}{0,972} & \multirow{4}{*}{0,65} & \multirow{4}{*}{74,45} & \multirow{4}{*}{82,23} & \multirow{4}{*}{$0,9224^{\mathrm{ns}}$} \\
\hline & $\beta_{1}$ & 1,96 & $4,83^{* *}$ & & & & & \\
\hline & $\beta_{2}$ & $-3,34.10^{-2}$ & $-5,17^{* *}$ & & & & & \\
\hline & $\beta_{3}$ & $1,97.10^{-4}$ & $5,94^{* *}$ & & & & & \\
\hline \multirow{4}{*}{3} & $\beta_{0}$ & $-64,77$ & $-0,61^{\mathrm{ns}}$ & \multirow{4}{*}{0,041} & \multirow{4}{*}{8,10} & \multirow{4}{*}{251,48} & \multirow{4}{*}{259,25} & \multirow{4}{*}{$0,9140^{* *}$} \\
\hline & $\beta_{1}$ & 3,83 & $0,74^{\mathrm{ns}}$ & & & & & \\
\hline & $\beta_{2}$ & $-6,19.10^{-2}$ & $-0,75^{\text {ns }}$ & & & & & \\
\hline & $\beta_{3}$ & $3,31.10^{-4}$ & $0,79^{\mathrm{ns}}$ & & & & & \\
\hline \multirow{4}{*}{4} & $\beta_{0}$ & $-71,97$ & $-13,68^{* *}$ & \multirow{4}{*}{0,993} & \multirow{4}{*}{0,48} & \multirow{4}{*}{72,35} & \multirow{4}{*}{81,70} & \multirow{4}{*}{$0,9769^{\mathrm{ns}}$} \\
\hline & $\beta_{1}$ & 4,12 & $15,77^{* *}$ & & & & & \\
\hline & $\beta_{2}$ & $-7,09.10^{-2}$ & $-17,07^{* *}$ & & & & & \\
\hline & $\beta_{3}$ & $4,09.10^{-4}$ & $19,25^{* *}$ & & & & & \\
\hline \multirow{4}{*}{5} & $\beta_{0}$ & $-61,01$ & $-11,31^{* *}$ & \multirow{4}{*}{0,988} & \multirow{4}{*}{0,47} & \multirow{4}{*}{65,07} & \multirow{4}{*}{74,00} & \multirow{4}{*}{$0,9810^{\text {ns }}$} \\
\hline & $\beta_{1}$ & 3,46 & $12,87^{* *}$ & & & & & \\
\hline & $\beta_{2}$ & $-5,87 \cdot 10^{-2}$ & $-13,68^{* *}$ & & & & & \\
\hline & $\beta_{3}$ & $3,35 \cdot 10^{-4}$ & $15,20^{* *}$ & & & & & \\
\hline \multirow{4}{*}{6} & $\beta_{0}$ & $-61,09$ & $-11,63^{* *}$ & \multirow{4}{*}{0,990} & & & & \\
\hline & $\beta_{1}$ & 3,42 & $13,17^{* *}$ & & 047 & 6007 & 7832 & $0.9786^{\mathrm{ns}}$ \\
\hline & $\beta_{2}$ & $-5,79.10^{-2}$ & $-14,06^{* *}$ & & $0,4 /$ & 69,07 & 18,32 & $0,9 / 86^{30}$ \\
\hline & $\beta_{3}$ & $3,31 \cdot 10^{-4}$ & $15,73^{* *}$ & & & & & \\
\hline
\end{tabular}

tc : t calculado; $\mathrm{R}^{2}$ ajust: coeficiente de determinação ajustado; AIC: critério de informação de Akaike; BIC: critério de informação Bayesiano; $\mathrm{S}_{\mathrm{xy}}$ : erro padrão da estimativa (\%); W: estimativa do parâmetro do teste de normalidade Shapiro-Wilk; **: significativo a $1 \%$; ns: não significativo a $5 \%$.

Observa-se que, com exceção do tratamento 3, os demais apresentaram similaridade para o comportamento da umidade de equilíbrio em função da umidade relativa do ar. Esse resultado é um indicativo de que houve diferença entre os tipos de adesivos utilizados, pois se observa que os painéis produzidos com o adesivo MUF, apresentaram os maiores valores de umidades de equilíbrio, além de uma maior variabilidade dos dados, o que influenciou nos ajustes dos modelos. Essa maior variabilidade pode ter ocorrido, pela menor resistência do adesivo MUF a ação da umidade ou, também, por algum efeito de cura do adesivo proporcionado por um pH mais baixo deste em relação aos demais (Tabela 2). Enquanto que não houve diferenças significativas na variação na umidade de equilíbrio entre os adesivos PMUF e FF, apresentando inclusive o mesmo padrão de variação em função do aumento da umidade relativa do ar.

Cerne, Lavras, v. 20, n. 1, p. 123-138, jan./mar. 2014 
Paul et al. (2006) estudando o efeito de diferentes tipos de adesivos na produção de painéis OSB, também observaram igualdade estatística entre as umidade de equilíbrio dos painéis produzidos com os adesivos PMUF e FF, sendo obtidos os valores médios de $9,5 \%$ e de $10,5 \%$, respectivamente, quando os painéis foram expostos a temperatura de $20^{\circ} \mathrm{C}$ e umidade relativa de $65 \%$.

Em relação à inclusão laminar observa-se que não houve diferença quanto ao tipo de adesivo utilizado na produção do painel OSB. Isso ocorreu, provavelmente, porque as lâminas foram coladas com o adesivo fenolformaldeído o que pode ter ocasionado uma barreira à penetração de umidade nos painéis produzidos com o adesivo MUF, fazendo com que ocorresse a diminuição significativa na umidade de equilíbrio dos painéis nas diferentes umidades relativas avaliadas. Os painéis com e sem inclusão laminar apresentaram o mesmo padrão de variação da umidade de equilíbrio quando produzidos com os adesivos PMUF e FF, demonstrando que a inclusão laminar não afetou a umidade de equilíbrio.
Silva et al. (2005), ao verificarem a umidade de equilíbrio de diferentes tipos de painéis de madeira, obtiveram para os painéis OSB sem revestimento valores variando entre 8,1 a $19,7 \%$ quando avaliada a amplitude de 40 a $90 \%$ de umidade relativa do ar.

Silva et al. (2006b), ao avaliarem a estimativa da umidade de equilíbrio de painéis de madeira mediante a utilização da equação de nelson obtiveram valores de umidade de equilíbrio entre 13,1 a $15,6 \%$ para os painéis OSB produzidos com madeira de coníferas. Valores estes próximos aos obtidos neste estudo, quando os painéis foram submetidos à faixa de umidade relativa entre 80 a $90 \%$. No entanto, deve-se levar em conta as diferentes especificidades de cada tipo de painel avaliado.

Os parâmetros dos modelos não lineares ajustados com as diferentes umidades relativas, considerando a temperatura média de $40^{\circ} \mathrm{C}$, bem como algumas estatísticas utilizadas na seleção dos melhores modelos são apresentados na Tabela 5.
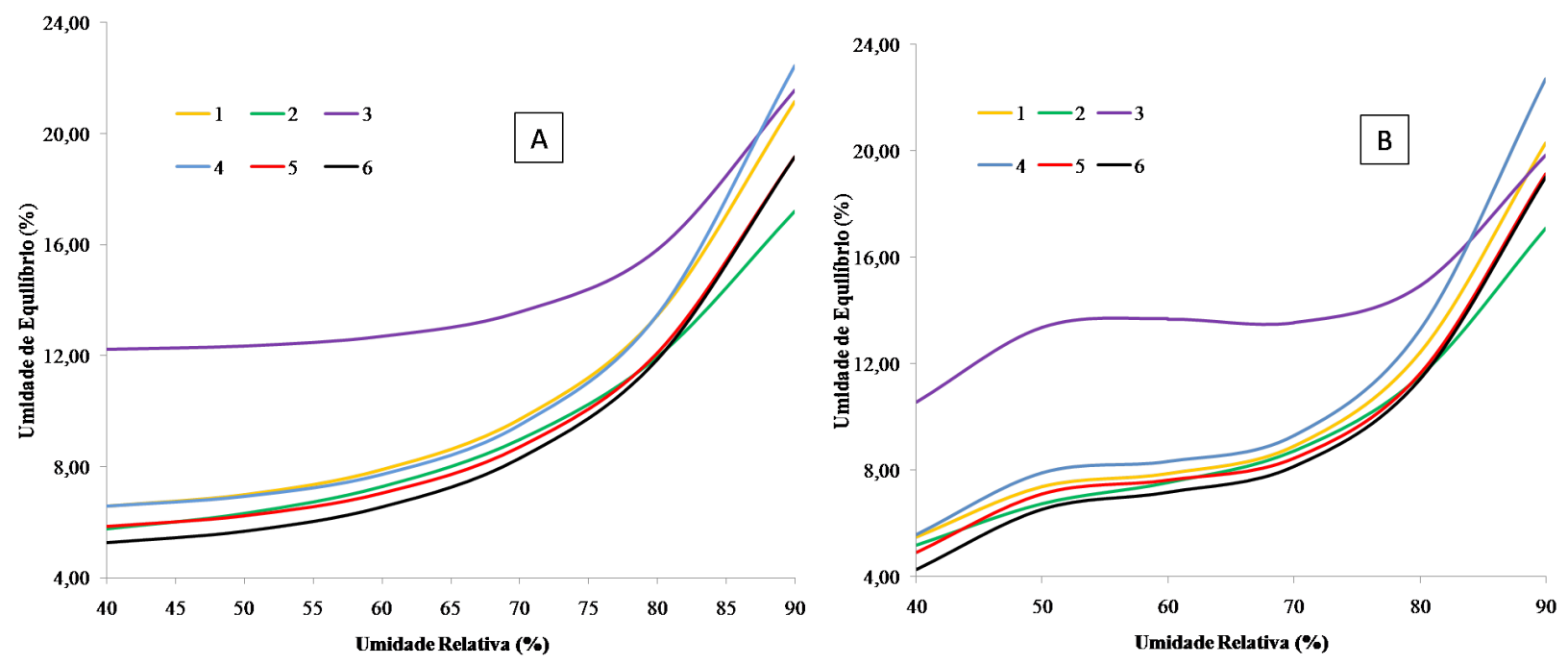

Figura 2 - Representação gráfica dos modelos não lineares (A) e polinomiais de terceira ordem (B) ajustados para os diferentes tratamentos para a temperatura de $30^{\circ} \mathrm{C}$.

Figure 2 - Graphical representation of nonlinear models $(A)$ and third-order polynomial $(B)$ adjusted for different treatments for the temperature of $30^{\circ} \mathrm{C}$.

Cerne, Lavras, v. 20, n. 1, p. 123-138, jan./mar. 2014 
Tabela 5 - Parâmetros dos modelos não lineares ajustados considerando a temperatura média de $40^{\circ} \mathrm{C}$.

Table 5 - Parameters of the nonlinear models adjusted considering the average temperature of $40^{\circ} \mathrm{C}$.

\begin{tabular}{|c|c|c|c|c|c|c|c|c|}
\hline Tratamentos & \multicolumn{2}{|c|}{ Coeficientes } & tc & $\mathrm{R}_{\text {ajust }}^{2}$ & $S_{x y}$ & AIC & $\mathrm{BIC}$ & $\mathrm{W}$ \\
\hline \multirow{3}{*}{1} & $\beta_{0}$ & 5,681 & $20,22^{* *}$ & & & & & \\
\hline & $\beta_{1}$ & 0,017 & $2,36^{*}$ & 0,998 & 0,84 & 124,25 & 131,74 & $0,9647^{\mathrm{ns}}$ \\
\hline & $\beta_{2}$ & 0,076 & $16,49^{* *}$ & & & & & \\
\hline \multirow{3}{*}{2} & $\beta_{0}$ & 4,809 & $12,68^{* *}$ & & & & & \\
\hline & $\beta_{1}$ & 0,043 & $1,83^{\mathrm{ns}}$ & 0,998 & 0,79 & 89,86 & 96,20 & $0,9690^{\text {ns }}$ \\
\hline & $\beta_{2}$ & 0,063 & $10,77^{* *}$ & & & & & \\
\hline \multirow{3}{*}{3} & $\beta_{0}$ & $-1,735$ & $-0,08^{\text {ns }}$ & & & & & \\
\hline & $\beta_{1}$ & 3,935 & $0,31^{\mathrm{ns}}$ & 0,884 & 6,16 & 205,58 & 211,32 & $0,9027^{* *}$ \\
\hline & $\beta_{2}$ & 0,021 & $0,77^{\mathrm{ns}}$ & & & & & \\
\hline \multirow{3}{*}{4} & $\beta_{0}$ & 6,008 & $22,89^{* *}$ & & & & & \\
\hline & $\beta_{1}$ & 0,014 & $2,40^{*}$ & 0,998 & 0,81 & 120,89 & 128,38 & $0,9822^{\mathrm{ns}}$ \\
\hline & $\beta_{2}$ & 0,078 & $17,26^{* *}$ & & & & & \\
\hline \multirow{3}{*}{5} & $\beta_{0}$ & 5,112 & $20,02^{* *}$ & & & & & \\
\hline & $\beta_{1}$ & 0,019 & $2,30^{*}$ & 0,998 & 0,68 & 83,97 & 90,52 & $0,9692^{\text {ns }}$ \\
\hline & $\beta_{2}$ & 0,073 & $15,45^{* *}$ & & & & & \\
\hline \multirow{3}{*}{6} & $\beta_{0}$ & 4,519 & $11,82^{* *}$ & & & & & \\
\hline & $\beta_{1}$ & 0,027 & $1,74^{\mathrm{ns}}$ & 0,997 & 1,02 & 134,72 & 141,95 & $0,8367^{* *}$ \\
\hline & $\beta_{2}$ & 0,069 & $11,14^{* *}$ & & & & & \\
\hline
\end{tabular}

tc : t calculado; R² ajust: coeficiente de determinação ajustado; AIC: critério de informação de Akaike; BIC: critério de informação Bayesiano; $\mathrm{S}_{\mathrm{xy}}$ : erro padrão da estimativa (\%); W: estimativa do parâmetro do teste de normalidade Shapiro-Wilk; **: significativo a $1 \%$; * significativo a $5 \%$; ns: não significativo a $5 \%$.

Observou-se, para os modelos não lineares ajustados para a temperatura média de $40^{\circ} \mathrm{C}$, resultados semelhantes aos obtidos para os modelos não lineares ajustados, considerando-se a temperatura média de $30^{\circ} \mathrm{C}$ (Tabela 3 ).

Verifica-se que não se obteve um bom ajuste do modelo não linear para o tratamento 3 , em razão da maior variabilidade dos valores médios de umidade de equilíbrio observados.

Para os tratamentos 2 e 6 , observa-se que o coeficiente $\beta_{1}$ foi não significativo a $5 \%$ pelo teste t e pode ser removido do modelo. $\mathrm{O}$ modelo não linear ajustado para o tratamento 6 não apresentou normalidade residual. Logo, esse resultado pode comprometer as inferências relacionadas a essa equação.

Cerne, Lavras, v. 20, n. 1, p. 123-138, jan./mar. 2014
Os parâmetros dos modelos polinomiais de terceira ordem ajustados com as diferentes umidades relativas, considerando a temperatura média de $40^{\circ} \mathrm{C}$, bem como algumas estatísticas utilizadas na seleção dos melhores modelos, encontra-se na Tabela 6. Todos os modelos polinomiais foram significativos pelo Teste $\mathrm{F}(\mathrm{p}<0,01)$.

Observa-se que para o tratamento 3 não foi possível obter um ajuste satisfatório do modelo polinomial considerado, assim como o observado na temperatura $30^{\circ} \mathrm{C}$. Isso se deve à maior variabilidade observada nos painéis produzidos apenas com o adesivo MUF, já explicado em parágrafo anterior.

Mediante a análise dos critérios de Akaike (AIC) e o bayesiano (BIC), recomenda-se a utilização dos modelos polinomiais de terceira ordem ajustados. No entanto, para os 
tratamentos 2 e 6 não foi encontrada normalidade residual mediante a utilização do teste Shapiro-Wilk, a 5\% de significância.

Os valores estimados pelos modelos ajustados considerando-se a temperatura de $40^{\circ} \mathrm{C}$ estão apresentados na
Figura 3. Nota-se, assim como na temperatura de $30^{\circ} \mathrm{C}$, houve apenas diferença entre a curva ajustada para o tratamento $3 \mathrm{em}$ relação às demais. Dessa forma, pode ser admitida a mesma explicação dada para a temperatura de $30^{\circ} \mathrm{C}$.

Tabela 6 - Parâmetros dos modelos polinomiais de terceira ordem ajustados considerando a temperatura média de $40^{\circ} \mathrm{C}$.

Table 6 - Parameters of the third-order polynomial models adjusted considering the average temperature of $40^{\circ} \mathrm{C}$.

\begin{tabular}{|c|c|c|c|c|c|c|c|c|}
\hline Tratamentos & \multicolumn{2}{|c|}{ Coeficientes } & tc & $\mathrm{R}_{\text {ajust }}^{2}$ & $S_{x y}$ & AIC & $\mathrm{BIC}$ & $\mathrm{W}$ \\
\hline \multirow{4}{*}{1} & $\beta_{0}$ & $-70,15$ & $-12,84^{* *}$ & \multirow{4}{*}{0,992} & \multirow{4}{*}{0,50} & \multirow{4}{*}{75,99} & \multirow{4}{*}{85,35} & \multirow{4}{*}{$0,9615^{\text {ns }}$} \\
\hline & $\beta_{1}$ & 3,97 & $14,63^{* *}$ & & & & & \\
\hline & $\beta_{2}$ & $-6.80 .10^{-2}$ & $-15,76^{* *}$ & & & & & \\
\hline & $\beta_{3}$ & $3,91.10^{-4}$ & $17,76^{* *}$ & & & & & \\
\hline \multirow{4}{*}{2} & $\beta_{0}$ & $-44,01$ & $-5,58^{* *}$ & \multirow{4}{*}{0,979} & \multirow{4}{*}{0,63} & \multirow{4}{*}{74,44} & \multirow{4}{*}{82,35} & \multirow{4}{*}{$0,9237^{*}$} \\
\hline & $\beta_{1}$ & 2,55 & $6,51^{* *}$ & & & & & \\
\hline & $\beta_{2}$ & $-4,33 \cdot 10^{-2}$ & $-6,97^{* *}$ & & & & & \\
\hline & $\beta_{3}$ & $2,52 \cdot 10^{-4}$ & $7,91^{* *}$ & & & & & \\
\hline \multirow{4}{*}{3} & $\beta_{0}$ & 6,13 & $0,074^{\text {ns }}$ & \multirow{4}{*}{0,458} & \multirow{4}{*}{6,28} & \multirow{4}{*}{207,58} & \multirow{4}{*}{214,75} & \multirow{4}{*}{$0,9042^{* *}$} \\
\hline & $\beta_{1}$ & $-9,88 \cdot 10^{-2}$ & $-0,024^{\mathrm{ns}}$ & & & & & \\
\hline & $\beta_{2}$ & $3,25.10^{-3}$ & $0,048^{\text {ns }}$ & & & & & \\
\hline & $\beta_{3}$ & $1,04.10^{-6}$ & $0,003^{\text {ns }}$ & & & & & \\
\hline \multirow{4}{*}{4} & $\beta_{0}$ & $-69,19$ & $12,04^{* *}$ & \multirow{4}{*}{0,992} & \multirow{4}{*}{0,53} & \multirow{4}{*}{80,78} & \multirow{4}{*}{90,13} & \multirow{4}{*}{$0,9638^{\mathrm{ns}}$} \\
\hline & $\beta_{1}$ & 3,94 & $13,83^{* *}$ & & & & & \\
\hline & $\beta_{2}$ & $-6,77 \cdot 10^{-2}$ & $-14,94^{* *}$ & & & & & \\
\hline & $\beta_{3}$ & $3,92.10^{-4}$ & $16,88^{* *}$ & & & & & \\
\hline \multirow{4}{*}{5} & $\beta_{0}$ & $-52,30$ & $-9,58^{* *}$ & \multirow{4}{*}{0,991} & \multirow{4}{*}{0,45} & \multirow{4}{*}{53,69} & \multirow{4}{*}{61,87} & \multirow{4}{*}{$0,9561^{\mathrm{ns}}$} \\
\hline & $\beta_{1}$ & 3,01 & $11,16^{* *}$ & & & & & \\
\hline & $\beta_{2}$ & $-5,17 \cdot 10^{-2}$ & $-12,11^{* *}$ & & & & & \\
\hline & $\beta_{3}$ & $3,00.10^{-4}$ & $13,80^{* *}$ & & & & & \\
\hline \multirow{4}{*}{6} & $\beta_{0}$ & $-63,42$ & $-7,19^{* *}$ & & & & & \\
\hline & $\beta_{1}$ & 3,509 & $8,03^{* *}$ & 0.976 & 0.79 & 11200 & 12103 & $0.7986^{* *}$ \\
\hline & $\beta_{2}$ & $-5,91 \cdot 10^{-2}$ & $-8,54^{* *}$ & & & & 121,03 & 0,1986 \\
\hline & $\beta_{3}$ & $3,36.10^{-4}$ & $9,53^{* *}$ & & & & & \\
\hline
\end{tabular}

tc : $t$ calculado; $\mathrm{R}^{2}$ ajust: coeficiente de determinação ajustado; AIC: critério de informação de Akaike; BIC: critério de informação Bayesiano; $\mathrm{S}_{x y}$ : erro padrão da estimativa (\%); W: estimativa do parâmetro do teste de normalidade Shapiro-Wilk; **: significativo a $1 \%$; : significativo a $5 \%$; ns: não significativo a $5 \%$.

Cerne, Lavras, v. 20, n. 1, p. 123-138, jan./mar. 2014 

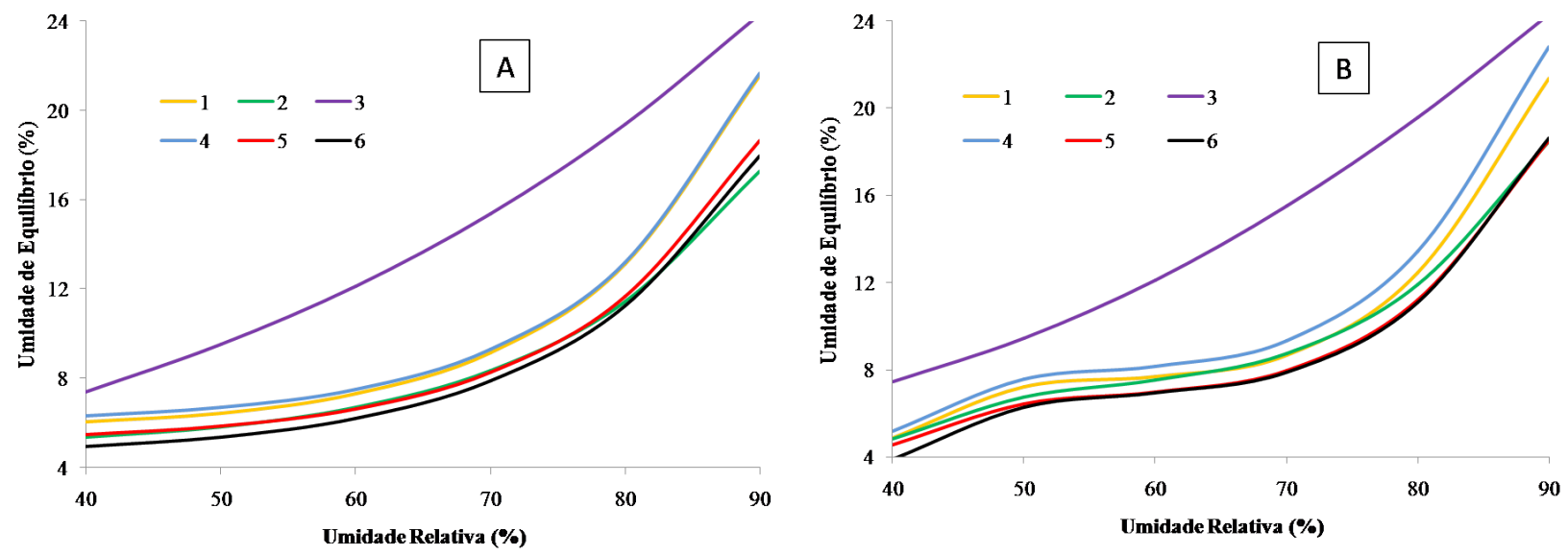

Figura 3 - Representação gráfica dos modelos não lineares (a) e polinomiais de terceira ordem (b) ajustados para os diferentes tratamentos para a temperatura de $40^{\circ} \mathrm{C}$.

Figure 3 - Graphical representation of nonlinear models (a) and third-order polynomial (b) adjusted for the different treatments for the temperature of $40^{\circ} \mathrm{C}$.

Na Tabela 7, estão apresentados os parâmetros dos modelos não lineares ajustados com as diferentes umidades relativas, considerando-se a temperatura média de $50^{\circ} \mathrm{C}$, assim como também algumas estatísticas utilizadas na avaliação dos modelos. Observa-se que todos os modelos ajustados apresentaram coeficientes significativos pelo teste t. Contudo, para o tratamento 3 não foi possível obter um bom ajuste do modelo não linear considerado, resultando em um baixo coeficiente de determinação ajustado, elevado erro padrão da estimativa e ausência de normalidade residual.

Tabela 7 - Parâmetros dos modelos não lineares ajustados considerando a temperatura média de $50^{\circ} \mathrm{C}$.

Table 7 -Parameters of the nonlinear models adjusted considering the average temperature of $50^{\circ} \mathrm{C}$.

\begin{tabular}{|c|c|c|c|c|c|c|c|c|}
\hline Tratamentos & \multicolumn{2}{|c|}{ Coeficientes } & tc & $\mathrm{R}_{\text {ajust }}^{2}$ & $\mathrm{~S}_{\mathrm{xy}}$ & AIC & $\mathrm{BIC}$ & $\mathrm{W}$ \\
\hline \multirow{2}{*}{1} & $\beta_{0}$ & 1,616 & $27,19^{* *}$ & \multirow{2}{*}{0,989} & \multirow{2}{*}{0,48} & \multirow{2}{*}{70,31} & \multirow{2}{*}{75,93} & \multirow{2}{*}{$0,9558^{\mathrm{ns}}$} \\
\hline & $\beta_{1}$ & 0,027 & $57,79^{* *}$ & & & & & \\
\hline \multirow{2}{*}{2} & $\beta_{0}$ & 1,677 & $15,06^{* *}$ & \multirow{2}{*}{0,966} & \multirow{2}{*}{0,68} & \multirow{2}{*}{78,05} & \multirow{2}{*}{82,80} & \multirow{2}{*}{$0,9152^{* *}$} \\
\hline & $\beta_{1}$ & 0,024 & $28,72^{* *}$ & & & & & \\
\hline \multirow{2}{*}{3} & $\beta_{0}$ & 3,396 & $2,54^{* *}$ & \multirow{2}{*}{0,411} & \multirow{2}{*}{6,31} & \multirow{2}{*}{206,16} & \multirow{2}{*}{210,46} & \multirow{2}{*}{$0,8631^{* *}$} \\
\hline & $\beta_{1}$ & 0,021 & $4,10^{* *}$ & & & & & \\
\hline \multirow{2}{*}{4} & $\beta_{0}$ & 1,701 & $30,32^{* *}$ & \multirow{2}{*}{0,990} & \multirow{2}{*}{0,42} & \multirow{2}{*}{57,86} & \multirow{2}{*}{63,48} & \multirow{2}{*}{$0,9693^{\mathrm{ns}}$} \\
\hline & $\beta_{1}$ & 0,025 & $61,21^{* *}$ & & & & & \\
\hline \multirow{2}{*}{5} & $\beta_{0}$ & 1,575 & $21,39^{* *}$ & \multirow{2}{*}{0,983} & \multirow{2}{*}{0,49} & \multirow{2}{*}{59,82} & \multirow{2}{*}{64,81} & \multirow{2}{*}{$0,9819^{\mathrm{ns}}$} \\
\hline & $\beta_{1}$ & 0,025 & $42,43^{* *}$ & & & & & \\
\hline \multirow{2}{*}{6} & $\beta_{0}$ & 1,381 & $20,98^{* *}$ & \multirow{2}{*}{0,980} & \multirow{2}{*}{0,50} & \multirow{2}{*}{71,91} & \multirow{2}{*}{77,46} & \multirow{2}{*}{$0,9688^{\text {ns }}$} \\
\hline & $\beta_{1}$ & 0,026 & $42,65^{* *}$ & & & & & \\
\hline
\end{tabular}

tc : t calculado; R² ajust: coeficiente de determinação ajustado; AIC: critério de informação de Akaike; BIC: critério de informação Bayesiano; $\mathrm{S}_{\mathrm{xy}}$ : erro padrão da estimativa (\%); W: estimativa do parâmetro do teste de normalidade Shapiro-Wilk; **: significativo a $1 \% ; *$ : significativo a $5 \%$; ns: não significativo a $5 \%$.

Cerne, Lavras, v. 20, n. 1, p. 123-138, jan./mar. 2014 
Na Tabela 8, encontram-se os parâmetros dos modelos polinomiais de segunda ordem ajustados com as diferentes umidades relativas, considerandose a temperatura média de $50^{\circ} \mathrm{C}$, assim como também algumas estatísticas utilizadas na avaliação dos modelos. Observou-se que todos os modelos polinomiais foram significativos pelo Teste $\mathrm{F}(\mathrm{p}<0,01)$.
Comparando-se os critérios de informação de Akaike e o critério de informação Bayesiano dos dois modelos ajustados para cada tratamento, recomendase a utilização dos polinômios de segundo grau para a estimativa da umidade de equilíbrio de painéis submetidos a condições de temperatura média do ar de $50^{\circ} \mathrm{C}$.

Tabela 8 - Parâmetros dos modelos polinomiais de segunda ordem ajustados considerando a temperatura média de $50^{\circ} \mathrm{C}$.

Table 8 -Parameters of the third-order polynomial models adjusted considering the average temperature of $50^{\circ} \mathrm{C}$.

\begin{tabular}{|c|c|c|c|c|c|c|c|c|}
\hline \multirow[t]{2}{*}{ Tratamentos } & \multicolumn{2}{|c|}{ Coeficientes } & \multirow{2}{*}{$\frac{\text { tc }}{6,24^{* *}}$} & \multirow[t]{2}{*}{$\mathrm{R}_{\text {ajust }}^{2}$} & \multirow[t]{2}{*}{$S_{x y}$} & \multirow[t]{2}{*}{ AIC } & \multirow[t]{2}{*}{ BIC } & \multirow[t]{2}{*}{ W } \\
\hline & $\beta_{0}$ & 7,4938 & & & & & & \\
\hline \multirow[t]{3}{*}{1} & $\beta_{1}$ & $-0,2089$ & $-5,38^{* *}$ & 0,987 & 0,51 & 76,94 & 84,43 & $0,9665^{\mathrm{ns}}$ \\
\hline & $\beta_{2}$ & 0,003599 & $12,14^{* *}$ & & & & & \\
\hline & $\beta_{0}$ & 6,1441 & $3,26^{* *}$ & & & & & \\
\hline \multirow[t]{3}{*}{2} & $\beta_{1}$ & $-0,1467$ & $-2,41^{*}$ & 0,964 & 0,69 & 80,82 & 87,15 & $0,9315^{*}$ \\
\hline & $\beta_{2}$ & 0,0027 & $5,82^{* *}$ & & & & & \\
\hline & $\beta_{0}$ & $-5,5250$ & $-0,305^{\text {ns }}$ & & & & & \\
\hline \multirow[t]{3}{*}{3} & $\beta_{1}$ & 0,3081 & $0,521^{\mathrm{ns}}$ & 0,378 & 6,38 & 207,73 & 213,46 & $0,8923^{* *}$ \\
\hline & $\beta_{2}$ & $-0,000088$ & $-0,019^{\text {ns }}$ & & & & & \\
\hline & $\beta_{0}$ & 9,5183 & $10,37^{* *}$ & & & & & \\
\hline \multirow[t]{3}{*}{4} & $\beta_{1}$ & $-0,2609$ & $-8,81^{* *}$ & 0,991 & 0,39 & 51,07 & 58,56 & $0,9855^{\text {ns }}$ \\
\hline & $\beta_{2}$ & 0,0038 & $16,86^{* *}$ & & & & & \\
\hline & $\beta_{0}$ & 6,7696 & $5,17^{* *}$ & & & & & \\
\hline \multirow[t]{3}{*}{5} & $\beta_{1}$ & $-0,1725$ & $-4,08^{* *}$ & 0,983 & 0,50 & 61,94 & 68,60 & $0,9739^{\text {ns }}$ \\
\hline & $\beta_{2}$ & 0,00289 & $9,00^{* *}$ & & & & & \\
\hline & $\beta_{0}$ & 4,3453 & $3,64^{* *}$ & & & & & \\
\hline \multirow[t]{2}{*}{6} & $\beta_{1}$ & $-0,1065$ & $-2,76^{* *}$ & 0,980 & 0,50 & 73,61 & 81,01 & $0,9609^{\text {ns }}$ \\
\hline & $\beta_{2}$ & 0,002347 & $7,97^{* *}$ & & & & & \\
\hline
\end{tabular}

tc : t calculado; $\mathrm{R}^{2}$ ajust: coeficiente de determinação ajustado; AIC: critério de informação de Akaike; BIC: critério de informação Bayesiano; $\mathrm{S}_{x y}$ : erro padrão da estimativa (\%); W: estimativa do parâmetro do teste de normalidade Shapiro-Wilk; **: significativo a $1 \%$; : significativo a $5 \%$; ns: não significativo a $5 \%$.

Na Figura 4, estão apresentados os modelos não lineares e polinomiais de segunda ordem ajustados para os diferentes tratamentos considerando-se a temperatura de $50^{\circ} \mathrm{C}$. Observa-se que os painéis submetidos à condição de temperatura de $50^{\circ} \mathrm{C}$ apresentaram comportamento médio distinto daqueles acondicionados sob temperatura média do ar de $30 \mathrm{e}$ $40^{\circ} \mathrm{C}$, tendo, assim, uma relação mais linear, ou seja, um aumento da umidade de equilíbrio dos painéis OSB com o aumento da umidade relativa do ar.

Cerne, Lavras, v. 20, n. 1, p. 123-138, jan./mar. 2014 

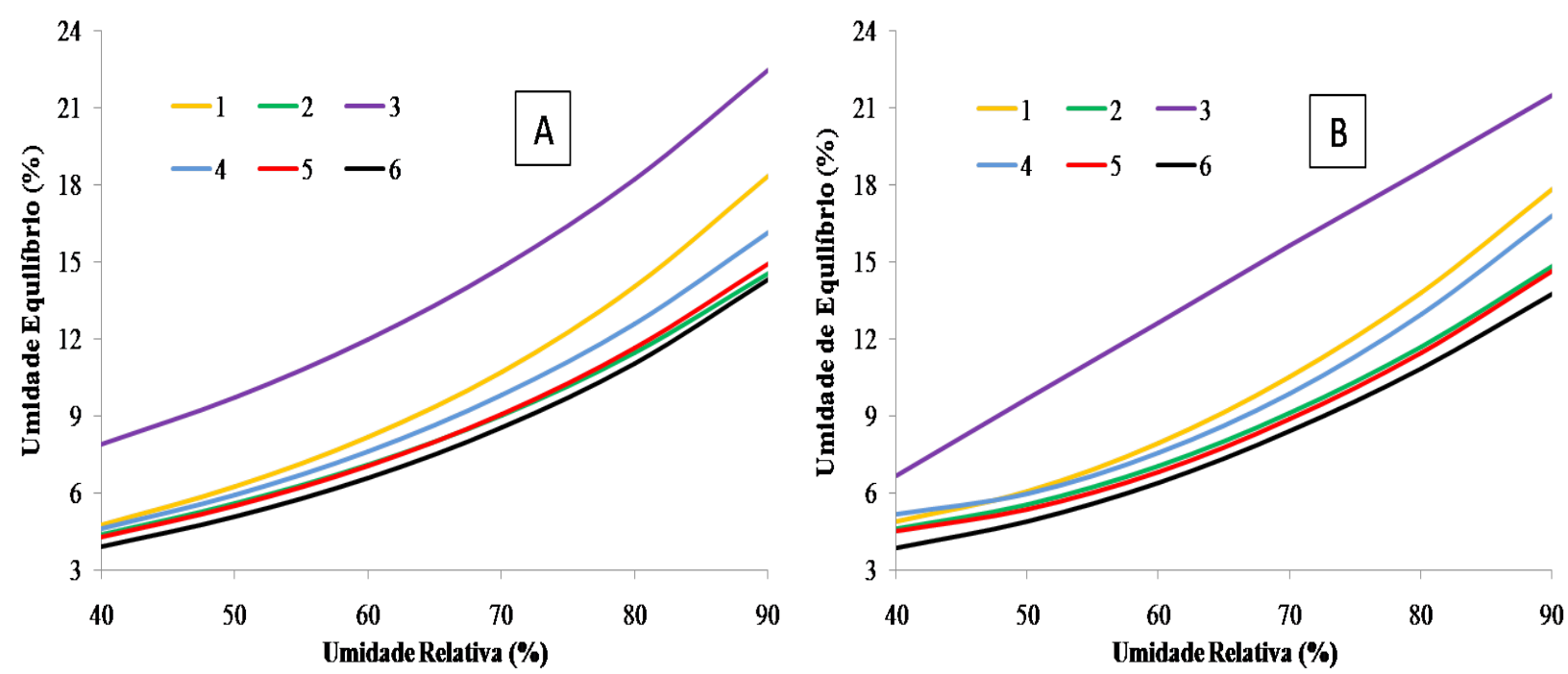

Figura 4 - Representação gráfica dos modelos não lineares (a) e polinomiais de segunda ordem (b) ajustados para os diferentes tratamentos considerando-se a temperatura de $50^{\circ} \mathrm{C}$.

Figure 4 - Graphical representation of nonlinear models (a) and third-order polynomial (b) adjusted for the different treatments for the temperature of $50^{\circ} \mathrm{C}$.

Na Tabela 9, encontram-se as superfícies de resposta (considerando como variáveis independentes a temperatura e umidade relativa do ar) ajustadas para os diferentes tratamentos e algumas estatísticas utilizadas na avaliação dos modelos.

Pode-se observar que as superfícies de resposta foram altamente significativas pelo Teste F. Contudo, para o tratamento 3 não foi encontrado um comportamento polinomial quadrático satisfatório, considerando-se a temperatura e umidade relativa do ar como variáveis independentes.

Para os demais tratamentos, obteve-se um alto coeficiente de determinação e baixo erro padrão da estimativa, sendo que apenas para o tratamento 2 não foi encontrada normalidade residual a $5 \%$ pelo teste de Shapiro-Wilk.

Apesar dos bons ajustes encontrados para os modelos que utilizaram a temperatura e umidade relativa do ar, observa-se que os modelos que utilizaram apenas a umidade relativa tiveram, em alguns casos, melhores ajustes e menores valores de AIC e BIC. Esse resultado evidência a possibilidade da estimativa da umidade de equilíbrio de painéis de madeira considerando-se apenas a umidade relativa do ar.

Apenas para o tratamento 2 foi observado um ponto de mínimo que resulta na umidade de equilíbrio de 4,53, tal resultado é obtido substituindo as regressões obtidas (Tabela 9) pelos valores de umidade relativa e temperatura da Tabela 10. Nota-se que alguns pontos estacionários estão fora da área experimental, sendo um indicativo da necessidade de novos ajustes.

\section{CONCLUSÕES}

De forma geral, os modelos polinomiais são os mais indicados para a determinação da umidade de equilíbrio dos painéis OSB. Sendo os modelos ajustados apenas com a umidade relativa do ar os que obtiveram melhores medidas de precisão.

O tipo de adesivo afetou a umidade de equilíbrio dos painéis, sendo observada para os adesivos PMUF e FF a mesma tendência de variação, e os maiores valores médios obtidos para os painéis produzidos com adesivo MUF.

A inclusão laminar promoveu a diminuição da umidade de equilíbrio apenas para os painéis produzidos com o adesivo MUF. 
Tabela 9 - Superfícies de resposta ajustadas para os diferentes tratamentos.

Table 9 - Surface Response adjusted for different treatments.

\begin{tabular}{|c|c|c|c|c|c|c|c|c|c|}
\hline Tratamentos & & ientes & tc & $\mathrm{Fc}$ & $\mathrm{R}_{\mathrm{aj}}^{2}$ & $S_{x y}$ & AIC & $\mathrm{BIC}$ & W \\
\hline \multirow{6}{*}{1} & $\beta_{0}$ & 14,13344 & $3,28^{* *}$ & \multirow{6}{*}{$478,7^{*}$} & \multirow{6}{*}{0,94} & \multirow{6}{*}{1,26} & \multirow{6}{*}{510} & \multirow{6}{*}{531} & \multirow{6}{*}{$0,985^{\text {ns }}$} \\
\hline & $\beta_{1}$ & $-0,45109$ & $-8,62^{* *}$ & & & & & & \\
\hline & $\beta_{2}$ & 0,05395 & $0,30^{\mathrm{ns}}$ & & & & & & \\
\hline & $\beta_{3}$ & $-0,00057$ & $-0,76^{\mathrm{ns}}$ & & & & & & \\
\hline & $\beta_{4}$ & 0,00581 & $17,22^{* *}$ & & & & & & \\
\hline & $\beta_{5}$ & $-0,00070$ & $-0,32^{\mathrm{ns}}$ & & & & & & \\
\hline \multirow{6}{*}{2} & $\beta_{0}$ & 13,28876 & $2,17^{*}$ & \multirow{6}{*}{$125,3^{*}$} & \multirow{6}{*}{0,84} & \multirow{6}{*}{1,59} & \multirow{6}{*}{463} & \multirow{6}{*}{485} & \multirow{6}{*}{$0,850^{* *}$} \\
\hline & $\beta_{1}$ & $-0,23287$ & $-3,17^{* *}$ & & & & & & \\
\hline & $\beta_{2}$ & $-0,19261$ & $-0,76^{\text {ns }}$ & & & & & & \\
\hline & $\beta_{3}$ & 0,00043 & $0,40^{\text {ns }}$ & & & & & & \\
\hline & $\beta_{4}$ & 0,00319 & $6,80^{* *}$ & & & & & & \\
\hline & $\beta_{5}$ & 0,00175 & $0,57^{\text {ns }}$ & & & & & & \\
\hline \multirow{6}{*}{3} & $\beta_{0}$ & 18,58666 & $0,63^{\text {ns }}$ & \multirow{6}{*}{$10,28^{* *}$} & \multirow{6}{*}{0,31} & \multirow{6}{*}{6,75} & \multirow{6}{*}{700} & \multirow{6}{*}{719} & \multirow{6}{*}{$0,933^{* *}$} \\
\hline & $\beta_{1}$ & $-0,35607$ & $-1,04^{\mathrm{ns}}$ & & & & & & \\
\hline & $\beta_{2}$ & $-0,07377$ & $-0,06^{\mathrm{ns}}$ & & & & & & \\
\hline & $\beta_{3}$ & 0,00780 & $1,61^{\text {ns }}$ & & & & & & \\
\hline & $\beta_{4}$ & 0,00233 & $1,08^{\mathrm{ns}}$ & & & & & & \\
\hline & $\beta_{5}$ & $-0,00540$ & $-0,37^{\mathrm{ns}}$ & & & & & & \\
\hline \multirow{6}{*}{4} & $\beta_{0}$ & 6,60219 & $1,40^{\mathrm{ns}}$ & \multirow{6}{*}{$413,1^{* *}$} & \multirow{6}{*}{0,93} & \multirow{6}{*}{1,38} & \multirow{6}{*}{536} & & \\
\hline & $\beta_{1}$ & $-0,40164$ & $-7,03^{* *}$ & & & & & & \\
\hline & $\beta_{2}$ & 0,38007 & $1,92^{\mathrm{ns}}$ & & & & & & \\
\hline & $\beta_{3}$ & $-0,00294$ & $-3,58^{* *}$ & & & & & 557 & $0,985^{1 \mathrm{si}}$ \\
\hline & $\beta_{4}$ & 0,00618 & $16,76^{* *}$ & & & & & & \\
\hline & $\beta_{5}$ & $-0,00321$ & $-1,35^{\mathrm{ns}}$ & & & & & & \\
\hline & $\beta_{0}$ & 7,83000 & $0,98^{\text {ns }}$ & & & & & & \\
\hline & $\beta_{1}$ & $-0,02602$ & $-2,76^{* *}$ & & & & & & \\
\hline 5 & $\beta_{2}$ & 0,01192 & $0,35^{\mathrm{ns}}$ & $1051^{* *}$ & & & & & $0507^{*}$ \\
\hline 5 & $\beta_{3}$ & $-3,219.10^{-3}$ & $-2,37^{*}$ & 105,1 & 0,80 & 2,16 & 590 & 610 & 0,597 \\
\hline & $\beta_{4}$ & $4,853.10^{-3}$ & $7,67^{* *}$ & & & & & & \\
\hline & $\beta_{5}$ & $9,064 \cdot 10^{-5}$ & $0,022^{\text {ns }}$ & & & & & & \\
\hline & $\beta_{0}$ & 0,58335 & $0,13^{\mathrm{ns}}$ & & & & & & \\
\hline & $\beta_{1}$ & $-0,25726$ & $-4,88^{* *}$ & & & & & & \\
\hline 6 & $\beta_{2}$ & 0,44983 & $2,45^{*}$ & $3597^{* *}$ & & 124 & & 513 & $0.986 \mathrm{~ns}$ \\
\hline 6 & $\beta_{3}$ & $-0,00272$ & $-3,62^{* *}$ & 359,1 & 0,92 & 1,24 & 492 & 313 & $0,986^{135}$ \\
\hline & $\beta_{4}$ & 0,00466 & $13,75^{* *}$ & & & & & & \\
\hline & $\beta_{5}$ & $-0,00425$ & $-1,93^{\text {ns }}$ & & & & & & \\
\hline
\end{tabular}

tc : t calculado; R² ajust: coeficiente de determinação ajustado; AIC: critério de informação de Akaike; BIC: critério de informação Bayesiano; $\mathrm{S}_{\mathrm{xy}}$ : erro padrão da estimativa (\%); W: estimativa do parâmetro do teste de normalidade Shapiro-Wilk; **: significativo a $1 \%$; *: significativo a $5 \%$; ns: não significativo a $5 \%$.

Cerne, Lavras, v. 20, n. 1, p. 123-138, jan./mar. 2014 
Tabela 10 - Coordenadas e classificação do ponto estacionáriodas superfícies de resposta ajustadas para cada tratamento.

Table 10 - Coordinates and classification of stationary point of response surfaces for each treatment.

\begin{tabular}{|c|c|c|c|}
\hline \multirow{2}{*}{ Tratamentos } & \multicolumn{2}{|c|}{ Pontos estacionários } & \multirow{2}{*}{ Classificação } \\
\hline & Umidade relativa (\%) & Temperatura $\left({ }^{\circ} \mathrm{C}\right)$ & \\
\hline 1 & 39,88 & 22,29 & Sela \\
\hline 2 & 33,07 & 50,85 & Mínimo \\
\hline 3 & 39,79 & 21,92 & Sela \\
\hline 4 & 42,03 & 39,96 & Sela \\
\hline 5 & 39,13 & 37,18 & Sela \\
\hline 6 & 39,33 & 40,35 & Sela \\
\hline
\end{tabular}

\section{REFERÊNCIAS}

AMERICAN SOCIETY FOR TESTING AND MATERIALS. ASTM D1582 : annual book of ASTM standards: adhesives. Washington, 1994.

MARTINS, V. A.; ALVES, M. V. S.; SILVA, J. F.; REBELLO, E. R. G.; PINHO, G.S.C. Umidade de equilíbrio e risco de apodrecimento da madeira em condições de serviço no Brasil. Brasil Florestal, Brasília, n. 76, p. 29-34, 2003.

MENDES, L. M.; ARCE, J. E. Análise comparativa das equações utilizadas para estimar a umidade de equilíbrio da madeira. Cerne, Lavras, v. 9, n. 2, p. 141-152, 2003.

MENDES, L. M.; CALEGÁRIO, N.; SILVA, G. A.; MELO, V. M.; LOPES, Y. L. V.; PÁDUA, F. A.; CARDOSO JUNIOR, A. A. C. Análise comparativa das equações utilizadas para estimar a umidade de equilíbrio da madeira: parte II. Brasil Florestal, Brasília, n. 79, p. 59-70, 2004.

MYERS, R. H. Response surface methodology. Boston: Allyn and Bacon, 1971. 246 p.

NELSON, R. M. A model for sorption of water vapor by cellulosic materials. Wood Fiber Science, Madison, v. 15, n. 1, p. 8-22, 1983.

PAUL, W.; OHLMEYER, M.; LEITHOFF, H.; BOONSTRA, M. J.; PIZZI, A. Optimising the properties of OSB by a one-step heat pre-treatment process. Holz als Roh-und Werkstoff, Berlin, v. 64, p. 227-234, 2006.
SILVA, G. A.; MENDES, L. M.; CALEGÁRIO, N.; MORI, F. A.; SILVA, J. R. M.; SANTOS, I. F. Estimativa da umidade de equilíbrio de painéis de madeira. Scientia Forestalis, Piracicaba, n. 70, p. 23-29, 2006b.

SILVA, G. A.; MENDES, L. M.; TRUGILHO, P. F.; MORI, F. A.; SANTOS, I. F. Umidade de equilíbrio de painéis de madeira. Revista Árvore, Viçosa, v. 29, n. 4, p. 639-646, jul./ago. 2005.

SILVA, G. A.; MENDES, L. M.; TRUGILHO, P. F.; MORI, F. A.; SANTOS, I. F.; PÁDUA, F. A. Efeito de algumas variáveis de processamento nas propriedades físicas de painéis de madeira de partículas alongadas. Ciência Florestal, Santa Maria, v. 16, n. 1, p. 51-60, 2006a.

SIMPSON, W. T. Equilibrium moisture content prediction for wood. Forest Products Journal, Madison, v. 21, n. 5, p. 48-49, 1971.

SIMPSON, W. T. Predicting equilibrium moisture content by mathematical models. Wood and Fiber, Madison, v. 5, n. 1, p. 41-45, 1973.

SIMPSON, W. T.; ROSEN, H. N. Equilibrium moisture content of wood at high temperatures. Wood and Fiber, Madison, v. 13, n. 3, p. 150-158, 1981.

SKAAR, C. Water in wood. New York: Syracuse University, 1972.

Cerne, Lavras, v. 20, n. 1, p. 123-138, jan./mar. 2014 
WERKEMA, C.; AGUIAR, S. Análise de regressão: como entender o relacionamento das variáveis de um processo. Belo Horizonte: Werkema, 2006. 306 p.

WU, Q. Application of Nelson's sorption isotherm to wood composites and overlays. Wood and Fiber Science, Madison, v. 28, n. 2, p. 227-239, 1999.

Recebido: 10 de agosto de 2011; aceito: 20 de agosto de 2013. 
